\title{
Pathological significance of connexin 26 expression in colorectal adenocarcinoma
}

\author{
RAN HONG ${ }^{1}$ and SUNG-CHUL LIM ${ }^{1,2}$ \\ ${ }^{1}$ Department of Pathology, ${ }^{2}$ Research Center for Resistant Cells, \\ College of Medicine, Chosun University, Gwangju, Korea
}

Received September 4, 2007; Accepted December 21, 2007

\begin{abstract}
This study was conducted to determine the level of expression and cellular localization of connexin 26 (Cx26) and the expression of p53 in colorectal adenocarcinoma as well as their relationship to clinicopathological features. Immunohistochemical staining was performed in 130 colorectal adenocarcinoma cases. A correlation between the expression levels of the two proteins and an analysis of the clinicopathological features of the samples was performed. There was a statistical significant difference in the Cx26 expression level among normal epithelium (NE), adenomas and adenocarcinomas $(\mathrm{p}<0.001)$. Of the 130 adenocarcinomas, $48.5 \%$ were positive for $\mathrm{Cx} 26$. All of the adenoma and NE samples were positive for $\mathrm{Cx} 26$ expression; however, the level of expression of $\mathrm{Cx} 26$ in adenomas was smaller than the level of expression for NE. Cytoplasmic staining for Cx26 was observed in the adenocarcinomas $(23.8 \%)$, but was not observed in the adenoma and NE samples. A positive correlation between a reduction in intercellular $\mathrm{Cx} 26$ and tumor invasion was statistically significant $(\mathrm{p}<0.05)$. Expression of p53 was positive for $50 \%$ of the adenocarcinomas, and the level of p53 was increased in a reverse proportion to the level of Cx26 intercellular staining. In conclusion, loss of intercellular and gain of intracytoplasmic Cx26 expression may play a role in colorectal carcinogenesis and tumor progression.
\end{abstract}

\section{Introduction}

One major cellular function is the maintenance of homeostasis. If impairment of the homeostatic state develops, various diseases, including malignant tumors, can develop. Gap junction intercellular communication (GJIC) controls proliferation, differentiation, migration, and other cellular

Correspondence to: Dr Sung-Chul Lim, Department of Pathology, Chosun University Hospital, 588 Seosuk-dong, Dong-gu, Gwangju 501-140, Korea

E-mail: sclim@chosun.ac.kr

Key words: colorectum, adenocarcinoma, connexin 26, immunohistochemistry functions and plays an important role in the maintenance of homeostasis and tumor suppression. Intercellular communication primarily is mediated through the gap junction, and minute water-soluble molecules (such as small metabolites, secondary messengers and inorganic ions) can migrate directly to the cytoplasm of adjacent cells (1).

The structural unit of the gap junction is the connexon, which is composed of six connexins $(\mathrm{Cx})$. The loss or dysfunction of GJIC or a mutation occurring in one of the connexin genes allows normal cells to evade normal growth control, resulting in their transformation to tumor cells (2-8). It has been previously reported that various types of malignant tumors, including skin, lung, and liver cancers (9-12), glioblastomas (13), and prostate cancers (14) are associated with the downregulation of $\mathrm{Cx}$ expression. Modified expression of $\mathrm{Cx}$, such as intracytoplasmic localization, has been reported to be associated with a malignant phenotype $(12,15,16)$. It has been shown that in cell lines defective in $\mathrm{Cx}$ expression, transfection of cDNA encoding connexin protein causes the recovery of the intercellular functional transmission system, resulting in the suppression of tumor growth $(17,18)$. As the gap junctions enable cell-cell communication and are involved in the suppression of tumor development, the Cx family of proteins can be considered tumor suppressors $(19,20)$.

It has been reported that in breast and ovarian cancer, as the clinical stage becomes more advanced, the expression of Cx26 is elevated (21). It has also been reported that Cx26 forms a heterologous gap junction with endothelial cells of the blood vessels in the vicinity of tumors, and thus facilitates the intravasation and extravasation of tumors and controls the invasion of the metastatic potential of tumor cells (22). Hence, it appears that the actual role of $\mathrm{Cx}$ and the gap junction may be different depending on tumor type and stage of progression.

In colorectal adenocarcinoma, it is known that the early loss and mutation of the $A P C$ gene appear prior to the formation of polyps. This accompanies the alteration of DNA methylation, and subsequently, in small-size adenomas, the $K$-ras gene is mutated. During the growth of an adenoma, the loss of the $18 \mathrm{q}$ chromosome arm occurs, and during the transition period from an adenoma to adenocarcinoma, tumor cells also undergo a sequential genetic change resulting in the mutation of the p53 gene (23). Therefore, a mutation of p53 is thought to play a major role in tumor progression, but not in the process of initiation of a colorectal adenocarcinoma. 
In the present study, through immunohistochemical methods, expression of Cx26 which is normally present on epithelial cells of the colorectum was evaluated. The specimens were classified as colorectal adenocarcinoma, adenoma, and normal epithelium, and the expression level of the protein as well as the expression pattern were evaluated. An association with the depth of invasion, nodal status, other organ metastasis, patient clinical stage, survival rate, survival length, and other previously known clinical factors related to prognosis was examined. Together with the status of p53 expression in colorectal adenocarcinoma, we characterized the role of $\mathrm{Cx} 26$ in tumorigenesis and in the progression of the cancer.

\section{Materials and methods}

Case selection and tissue sampling. One hundred and thirty subjects were chosen from patients diagnosed with colorectal adenocarcinoma who underwent surgery for the removal of tumors at the Department of Surgery, Chosun University Hospital from January 1992 to December 2004. Patients were selected in a non-consecutive manner when survival and the date of death could be assessed, when paraffin-embedded tissue storage conditions were adequate and when complete medical records were obtainable. In addition, for comparative analysis, 12 cases of colon adenoma that were obtained by endoscopic resection and 10 cases of normal mucosa obtained by endoscopic biopsy without specific histological abnormalities were included. In the selection of subjects, patients that received chemotherapeutic or radiation therapy prior to surgery, patients that received emergency surgery, or patients with hereditary non-polyposis colon cancer or with proof of familial adenomatous polyposis were excluded. Informed consent was obtained from each subject according to institutional guidelines, and the research protocol was approved by the Ethics Committee of Chosun University Hospital.

\section{Histopathological analysis}

Microscopic examination. Each tumor was re-examined retrospectively by analyzing the medical record and tissue slide of the patient. Age, gender, the histological pattern, the depth of invasion of the tumor, the presence or absence of metastasis to the lymph node or other organs, the expression of Cx26 as well as p53 protein were assessed. The tumor stages were determined according to the TNM staging system of the American Joint Committee on Cancer (24). The tissue samples to be observed were fixed in $10 \%$ neutral-buffered formalin, and the prepared paraffin-embedded tissues were sectioned $4-5 \mathrm{~mm}$ in thickness. Hematoxylin and eosion (H\&E) staining was performed, and the slides were examined under a light microscope.

Immunohistochemical analysis. All the samples investigated in this study were tested using goat polyclonal Cx26 antibody (N-19) (Santa Cruz Biotechnology, Santa Cruz, CA, USA) and mouse monoclonal p53 protein antibody (DO-7) (Dako, Glostrup, Denmark). Immunolocalization was performed using the ImmunoCruz ${ }^{\text {TM }}$ Staining System (Santa Cruz Biotechnology), according to the manufacturer's protocol. Briefly, 4- $\mu \mathrm{m}$ sections obtained after formalin fixation and paraffin embedding were deparaffinized in xylene and rehydrated with distilled water through a graded series of ethanol solutions. The sections were then placed in a glass jar with $10 \mathrm{mM}$ citrate buffer ( $\mathrm{pH} 6.0$ ), and irradiated in a microwave oven for $15 \mathrm{~min}$. The sections were allowed to cool in the jar at room temperature for $20 \mathrm{~min}$. The slides were then rinsed with Tris-buffered saline (TBS). Blocking reagent was added for $10 \mathrm{~min}$ after quenching of the endogenous peroxidase activity in $0.3 \%$ hydrogen peroxide for $10 \mathrm{~min}$. The slides were then washed as described above, and they were subsequently subjected to the primary antibody reaction. Cx26 (dilution 1:200) or p53 protein (dilution 1:100) was applied to the tissue sections, and they were allowed to incubate in a moist chamber overnight at $4^{\circ} \mathrm{C}$. After washing with TBS, a biotinylated antibody was added for $10 \mathrm{~min}$, followed by the addition of streptavidin peroxidase for an additional $10 \mathrm{~min}$. After washing out the excess complex, the localization of antibodies was visualized by incubating the sections for $15 \mathrm{~min}$ in the UltraVision Plus Detection System (Lab Vision, Fremont, CA, USA), and counterstaining was performed with Mayer's hematoxylin. The positive control for Cx26 was normal colon mucosa. Instead of the primary antibody, TBS was used as a negative control.

Analysis and interpretation of the immunohistochemical stains. Two pathologists who were blind to the clinical outcome of the samples performed an evaluation of the staining results. The result of $\mathrm{Cx} 26$ staining was classified as intercellular staining and intracytoplasmic staining, and regardless of the staining pattern, the percentage of positive tumor cells was determined, and depending on the staining level, reclassified as negative $(<10 \%$ positive) and positive ( $\geq 10 \%$ positive) (25).

Regarding intracytoplasmic staining, when a positive reaction in the cytoplasm was distinctly detected, it was considered to be positive. Concerning the p53 protein, nuclear staining was considered to be reactive, and the area showing a positive result was expressed as a percentage, and, according to the intensity of the staining, was classified as $0(0-9 \%), 1+$ $(10-39 \%), 2+(40-69 \%)$, and 3+ (70-100\%).

Statistical analysis. Information concerning patient survival was obtained from the hospital records and from the results of a survey conducted by the National Statistical Office. The mean follow-up time was 115 months (range, 38.2-155). For statistical analysis of the association of the expression of Cx26 as well as p53 protein and the various clinicopathological parameters, the $\chi^{2}$ test, Fisher's exact test, ANOVA, and the Student's t-test were applied. The level of significance was set at $\mathrm{p}<0.05$ for all cases. For statistical analysis, the Stat View software package (Abacus Concepts, Berkeley, CA, USA) was used.

\section{Results}

Clinical and histopathological findings. There were 62 male and 68 female patients (male:female ratio, 1:1.1). There were 32 cases $(24.6 \%)$ of patients 50-59 years of age, 46 cases $(35.4 \%)$ of patients $60-69$ years of age, and 29 cases $(22.3 \%)$ of patients $70-79$ years of age. The stages of the 
Table I. Relation between Cx26 immunoreactivity and the clinicopathologic features of the adenocarcinomas of the colorectum $(\%)$.

\begin{tabular}{|c|c|c|c|c|c|}
\hline & \multirow[t]{2}{*}{ No. } & \multicolumn{2}{|c|}{ Intercellular staining } & \multicolumn{2}{|c|}{ Cytoplasmic staining } \\
\hline & & + & - & + & - \\
\hline \multicolumn{6}{|l|}{ Gender } \\
\hline M & 62 & $25(40.3)$ & $37(59.7)$ & $15(24.2)$ & $47(75.8)$ \\
\hline $\mathrm{F}$ & 68 & $25(36.8)$ & $43(63.2)$ & $16(23.5)$ & $52(76.5)$ \\
\hline \multicolumn{6}{|l|}{ Age } \\
\hline $30-39$ & 5 & $1(20)$ & $4(80)$ & $2(40)$ & $3(60)$ \\
\hline $40-49$ & 13 & $4(30.8)$ & $9(69.2)$ & $1(7.7)$ & $12(92.3)$ \\
\hline $50-59$ & 32 & $13(40.6)$ & $19(59.4)$ & $11(31.3)$ & $21(65.6)$ \\
\hline $60-69$ & 46 & $19(41.3)$ & $27(58.7)$ & $11(23.9)$ & $35(76.1)$ \\
\hline $70-79$ & 29 & $11(37.9)$ & $18(62.1)$ & $5(17.2)$ & $24(82.8)$ \\
\hline $80-89$ & 5 & $2(40)$ & $3(60)$ & $1(20)$ & $4(80)$ \\
\hline \multicolumn{6}{|l|}{ T stage ${ }^{a}$} \\
\hline 1 & 5 & $3(60)$ & $2(40)$ & $1(20)$ & $4(80)$ \\
\hline 2 & 25 & $13(52)$ & $12(48)$ & $4(16)$ & $21(84)$ \\
\hline 3 & 97 & $34(35.1)$ & $63(64.9)$ & $24(24.7)$ & $73(75.3)$ \\
\hline 4 & 3 & 0 & $3(100)$ & $2(33.3)$ & $1(66.7)$ \\
\hline \multicolumn{6}{|l|}{$\mathrm{N}$ stage } \\
\hline 0 & 86 & 34 (39.5) & $52(60.5)$ & $21(24.4)$ & 65 (75.6) \\
\hline 1 & 41 & $16(39)$ & $25(61)$ & $7(17.1)$ & 34 (82.9) \\
\hline 2 & 3 & 0 & $3(100)$ & $2(33.3)$ & $1(66.7)$ \\
\hline \multicolumn{6}{|l|}{ M stage } \\
\hline 0 & 127 & 49 (38.6) & $78(61.4)$ & $29(22.8)$ & $98(77.2)$ \\
\hline 1 & 3 & $1(33.3)$ & $2(66.7)$ & $2(33.3)$ & $1(66.7)$ \\
\hline \multicolumn{6}{|c|}{ Clinical stage } \\
\hline I & 30 & 17 (56.7) & $13(43.3)$ & $6(20)$ & $24(80)$ \\
\hline II & 56 & $14(25)$ & $42(75)$ & $16(28.6)$ & 40 (71.4) \\
\hline III & 41 & $19(46.3)$ & $22(53.7)$ & $7(17.1)$ & 34 (82.9) \\
\hline IV & 3 & 0 & $3(100)$ & $2(33.3)$ & $1(66.7)$ \\
\hline
\end{tabular}

${ }^{a}$ Statistically significant difference between loss of intercellular Cx26 expression and $\mathrm{T}$ stage, $\mathrm{p}<0.05$.

tumors ( $\mathrm{T}, \mathrm{N}$ and $\mathrm{M}$ ) and the clinical stages of the 130 cases are listed in Table I.

\section{Clinicopathologic significance of Cx26 expression}

Positive cell rate of Cx26. Immunohistochemical staining for Cx26 was performed, and the number of cells showing positive expression of all of the tumor cells was expressed as a percentage (the positive cell rate). Only cases showing a $>10 \%$ positive cell rate were determined to be positive, and the association between the clinicopathological parameters and the differences between different cell types were compared and analyzed. Of the 130 adenocarcinoma cases, 63 cases (48.5\%) were Cx26 positive (Fig. 1) and 67 cases (51.5\%) were negative. For adenoma and normal mucosa cases, 12 out of 12 adenomas were Cx26 positive (Fig. 2) and 10 out of 10 normal mucosa were $\mathrm{Cx} 26$ positive; the level of $\mathrm{Cx} 26$ positive expression for all of the cell types was statistically

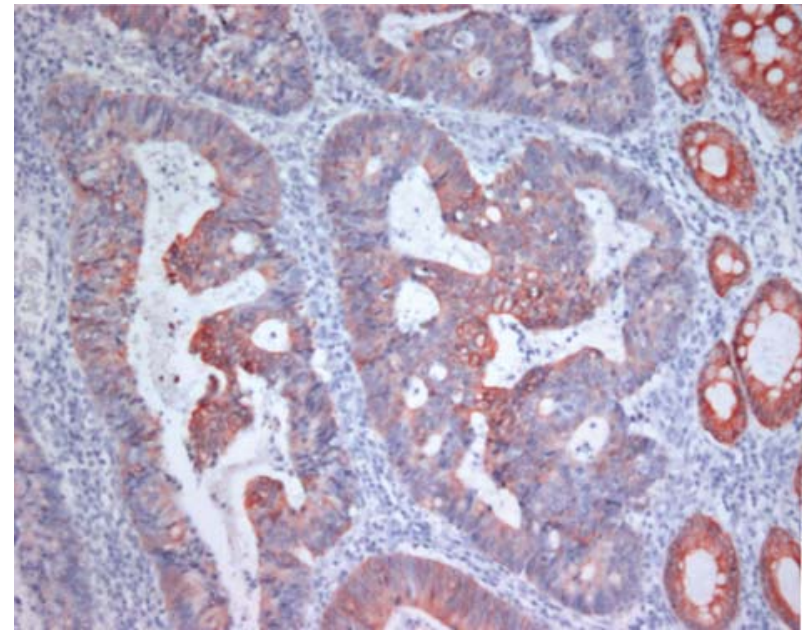

Figure 1. Immunohistochemical staining for $\mathrm{Cx} 26$ in a colonic adenocarcinoma. A decrease in intercellular Cx26 expression is noted in the adenocarcinoma (left) as compared with the normal epithelium (right) (LSAB method, counterstained with hematoxylin).

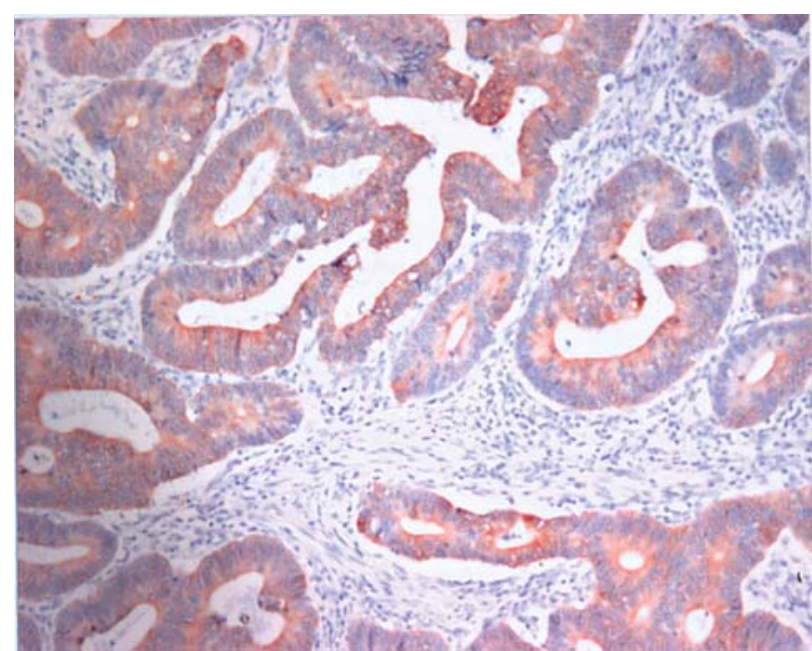

Figure 2. Immunohistochemical staining for $\mathrm{Cx} 26$ in a colonic adenoma. Intercellular immunoreactivity is identified but significantly decreased in the adenoma with severe dysplasia (LSAB method, counterstained with hematoxylin).

significant $(\mathrm{p}<0.0001)$ (Table II). The positive cell rate of adenocarcinomas showed a distribution of $0-80 \%$, and the mean positive cell rate was $18.6 \%$. Regarding the positive cell rate of Cx26 without distinguishing for intercellular staining and intracytoplasmic staining, the various clinicopathological parameters such as the depth of invasion, the nodal status, the presence or absence of distant metastasis, the survival length, and the tumor stage did not show a statistical significant association.

Intercellular staining of Cx26. For intercellular staining of $\mathrm{Cx} 26$, for the 130 adenocarcinoma cases, 50 cases (38.5\%) were determined to be positive and 80 cases $(61.5 \%)$ were negative; however, a significant difference according to age or gender was not found. Depth of invasion, nodal status, distant metastasis, and the clinical stage of the adenocarcinoma and intercellular staining of Cx26 were compared. 
Table II. Difference in Cx26 immunoreactivity among the normal mucosae, adenomas and adenocarcinomas of the colorectum $(\%)$.

\begin{tabular}{|c|c|c|c|c|c|}
\hline & \multirow[t]{2}{*}{ No. } & \multicolumn{2}{|c|}{ Immunoreactivity } & \multicolumn{2}{|c|}{ Cytoplasmic staining } \\
\hline & & + & - & + & - \\
\hline Normal mucosae & 10 & $10(100)$ & 0 & 0 & $10(100)$ \\
\hline Adenomas & 12 & $12(100)$ & 0 & 0 & $12(100)$ \\
\hline Adenocarcinomas & 130 & $63(48.5)$ & $67(51.5)$ & $31(23.8)$ & $99(76.2)$ \\
\hline p-value & & \multicolumn{2}{|c|}{$<0.0001$} & \multicolumn{2}{|c|}{$<0.05$} \\
\hline
\end{tabular}

Table III. Relation between clinicopathologic features and immunoreactivity of $\mathrm{p} 53$ protein in the colorectal adenocarcinomas $(\%)$.

\begin{tabular}{|c|c|c|c|c|c|}
\hline & \multirow[t]{2}{*}{ No. } & \multicolumn{4}{|c|}{ p53 protein immunoreactivity } \\
\hline & & 0 & 1 & 2 & 3 \\
\hline \multicolumn{6}{|c|}{ T stage } \\
\hline 1 & 5 & $3(60)$ & $1(20)$ & $1(20)$ & 0 \\
\hline 2 & 25 & $13(52)$ & $1(4)$ & $6(24)$ & $5(20)$ \\
\hline 3 & 97 & $49(50.5)$ & $11(11.3)$ & $23(23.7)$ & $14(14.4)$ \\
\hline 4 & 3 & 0 & 0 & 0 & $3(100)$ \\
\hline \multicolumn{6}{|c|}{$\mathrm{N}$ stage } \\
\hline 0 & 86 & $45(52.3)$ & $10(11.6)$ & $19(22.1)$ & $12(14)$ \\
\hline 1 & 41 & $20(48.8)$ & $3(7.3)$ & $11(26.8)$ & $7(17.1)$ \\
\hline 2 & 3 & 0 & 0 & 0 & $3(100)$ \\
\hline \multicolumn{6}{|c|}{ M stage } \\
\hline 0 & 127 & $65(51.6)$ & $13(103)$ & $30(23.8)$ & $19(14.9)$ \\
\hline 1 & 3 & 0 & 0 & 0 & $3(100)$ \\
\hline \multicolumn{6}{|c|}{ Clinical stage } \\
\hline I & 30 & $19(63)$ & $4(13.3)$ & $4(13.3)$ & $3(10)$ \\
\hline II & 56 & $29(51.8)$ & $7(12.5)$ & $14(25)$ & $6(10.7)$ \\
\hline III & 41 & $17(41.5)$ & $2(4.9)$ & $12(29.3)$ & $10(24.4)$ \\
\hline IV & 3 & 0 & 0 & 0 & $3(100)$ \\
\hline
\end{tabular}

The loss of Cx26 with invasion to the mucosa was detected in 2 of 5 cases $(40 \%)$, invasion to the muscle layer was detected in 12 of 25 cases $(48 \%)$, invasion to the pericolic adipose tissue was detected in 63 of 97 cases (64.9\%), and involvement of another organ was detected in 3 of 3 cases $(100 \%)$. A statistically significant difference for the depth of invasion of the tumors ( $\mathrm{pT})$ was observed $(\mathrm{p}=0.048)$. The low number of T1, N2 and M1 cases made it difficult to interpret and generalize the results. It is possible that the loss of Cx26 is associated with the progression of an adenocarcinoma, particularly for invasion of the tumors. However, the expression level of $\mathrm{Cx} 26$ according to lymph node metastasis $(\mathrm{pN})$, metastasis to other organs (pM), and clinical stage did not demonstrate a significant difference (Table I). In the follow-up examination of patients, an association between the survival rate and the intercellular expression of Cx26 was not statistically significant.

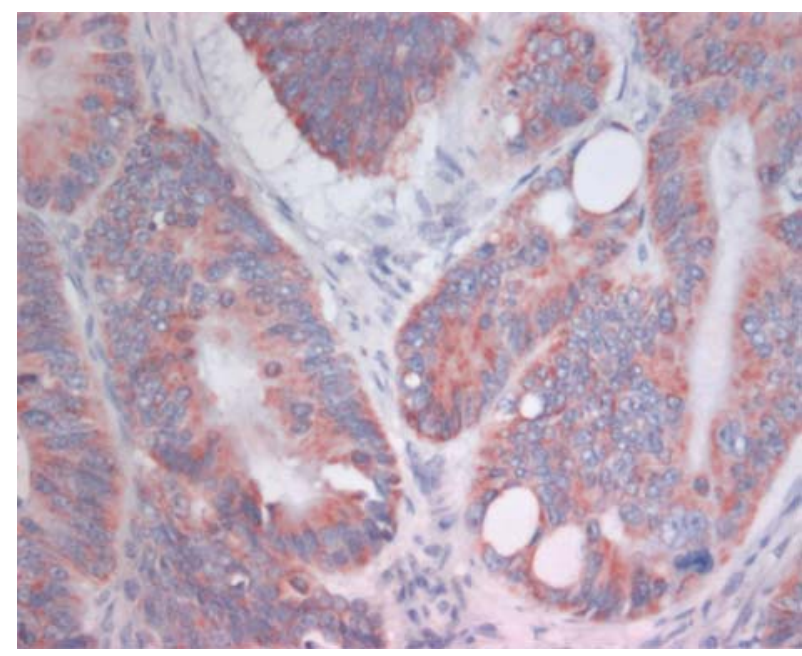

Figure 3. Immunohistochemical staining for $\mathrm{Cx} 26$ in a colonic adenocarcinoma. Distinct cytoplasmic immunoreactivity is noted. (LSAB method, counterstained with hematoxylin).

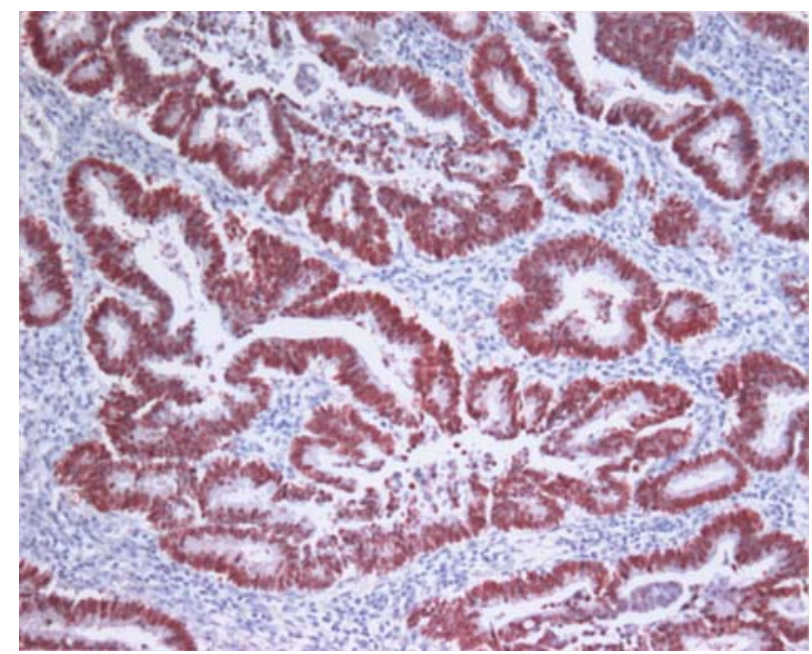

Figure 4. Immunohistochemical staining for p53 in a colonic adenocarcinoma. A strong nuclear staining is noted. (LSAB method, counterstained with hematoxylin).

Intracytoplasmic staining of Cx26. Intracytoplasmic staining was confirmed in 31 adenocarcinoma cases (23.8\%) (Fig. 3). For all of the adenoma cases and the normal epithelium cases, intracytoplasmic staining was not detected, and thus a significant difference among the cell types was detected $(p<0.001)$ (Table II). Gender, age, tumor invasion depth $(\mathrm{pT})$, lymph node metastasis $(\mathrm{pN})$, metastasis to other organs (pM), clinical stage, and other clinicopathological parameters did not show a correlation to the status of Cx26 intracytoplasmic staining (Table I). In the follow-up examination of patients, an association between patient survival rate and $\mathrm{Cx} 26$ intracytoplasmic staining was not statistically significant.

Clinicopathologic significance of p53 protein expression. Expression of p53 protein was found to be positive in 65 adenocarcinoma cases $(50 \%)$ and negative in 65 cases $(50 \%)$. 
Table IV. Relation between Cx26 immunoreactivity and expression of p53 protein in the colorectal adenocarcinomas (\%).

\begin{tabular}{|c|c|c|c|c|c|}
\hline & & \multicolumn{2}{|c|}{ Cx26 intercellular staining ${ }^{\mathrm{a}}$} & \multicolumn{2}{|c|}{ Cx26 cytoplasmic staining ${ }^{\mathrm{b}}$} \\
\hline & & + & - & + & - \\
\hline \multirow[t]{4}{*}{ p53 } & 0 & $29(44.6)$ & $36(55.4)$ & $11(16.9)$ & $54(83.1)$ \\
\hline & 1 & $5(38.5)$ & $8(61.5)$ & $4(30.8)$ & $9(69.2)$ \\
\hline & 2 & $10(33.3)$ & $20(66.7)$ & $9(30.0)$ & $21(70.0)$ \\
\hline & 3 & $6(27.3)$ & $16(72.7)$ & $7(31.8)$ & $15(68.2)$ \\
\hline
\end{tabular}

${ }^{\mathrm{a}} \mathrm{p}<0.05,{ }^{\mathrm{b}} \mathrm{p}>0.05$.

Expression was classified according to the staining intensity: 13 cases $(10 \%)$ were weakly positive $(1+), 30$ cases $(23.1 \%)$ were moderately positive $(2+)$ and 22 cases $(16.9 \%)$ were strongly positive (3+) (Fig. 4). The $\mathrm{N}$ stage, M stage and clinical stage dependent on the staining intensity of p53 had a positive correlation, but were not statistically significant because of the limited number of samples (Table III).

Comparing the expression level of $\mathrm{Cx} 26$ and p53 protein, it was found that in the cases which lost intercellular expression of Cx26, p53 protein was expressed more strongly, and thus a difference among the different case groups was observed ( $\mathrm{p}=0.035)$ (Table IV). The association between the survival rate of patients and expression of p53 protein was not statistically significant.

\section{Discussion}

The gap junction is a specialized cell membrane channel that mutually connects the cytoplasm of adjacent cells. Its structural unit is a connexon, and it is a hexameric hemichannel composed of six Cxs (26). GJIC is involved in the growth and differentiation of cells and thus it plays a role in maintaining homeostasis of tissues (27). It is possible that the intercellular exchange of molecules involved in the growth and death of cells may occur through the gap junctions (28).

The connection of the gap junction is known to decrease with the progression of the level of malignancy of tumor cells. A loss of Cx expression and the deterioration of the gap junction channel in tumors have been confirmed, and the overexpression of each $\mathrm{Cx}$ isoform could suppress the metastatic potential of cells. Thus, $\mathrm{Cx}$ can be considered a tumor suppressor. The traditional gap junction connection is formed by homotypic connexons, and this connexon is composed of one type of Cx. Generally, in the carcinogenic process, reduction in $\mathrm{Cx}$ expression or a reduction in gap junction function are related, and the overexpression of $\mathrm{Cx}$ suppresses tumors, which implies the role of the homotypic gap junction which is composed of the same type of connexon $(6,29)$. However, as tumors progress, Cx protein and the gap junction reappear. A connexon composed of heterogeneous Cx subtypes partly forms at the gap junction, resulting in a heterotypic gap junction. It has been reported that depending on the expression level of Cx subtypes, an abnormal cell transduction system between stromal cells of the host and the cancer cells is formed and induces phenotypic transformation of the host stromal cells resulting in the induction of the invasion of cancer cells $(22,30)$. In addition, it has been speculated that by controlling the expression of genes acting on the growth and differentiation of cells, $\mathrm{Cx}$ controls the progression of tumors (25). In a study by Ito et al (22), Cx controled the metastatic potential of malignant melanoma. The study suggests that the heterotypic gap junction between vascular endothelial cells and melanoma is involved in metastasis, and the expression of $\mathrm{Cx}$ of a heterotypic gap junction accelerates stromal invasion or even facilitates metastasis. Regarding Cx26 expression in the cytoplasm during the carcinogenic process of colorectal adenocarcinoma, it is speculated that the altered type of Cx protein is present in the tumor cell cytoplasm due to a transcriptional or posttranscriptional defect of the Cx protein (25). It is possible that in the cytoplasm of the neoplastic cells, an altered form of the Cx26 protein exists. Krutovskikh et al (31) confirmed that $\mathrm{Cx} 43$ was expressed on the cell membrane of normal colorectal epithelial cells together with Cx26 and Cx32, and due to a mutation in the $3 \mathrm{M}$ domain of $\mathrm{Cx} 43$, instead of being present between cells, Cx protein was present within cells. Thus, Cx protein is present within the nucleus and the cytoplasm. In addition, it is thought that $\mathrm{Cx} 43$ present in the nucleus and cytoplasm of tumor cells plays a role in controlling cell growth. Olbina and Eckhart (32) reported that due to the mutation of $\mathrm{Cx} 43$, the migration of this protein to the cell membrane is suppressed; nevertheless, it could not reduce the ability of $\mathrm{Cx}$ to suppress tumor cell growth. It was confirmed that the control of cell growth by $\mathrm{Cx}$ does not always require GJIC. Therefore, it is speculated that $\mathrm{Cx}$ protein expressed in the cytoplasm and $\mathrm{Cx}$ protein expressed between cells each play a different role in cell-activated signal transduction (25). However, the role of $\mathrm{Cx}$ in the signal transduction system requires further clarification and additional functional studies.

In this study, expression of $\mathrm{Cx} 26$ was found to be substantially reduced in adenocarcinomas as compared to adenomas and this reduction was statistically significant. In a study by Kanczuga-Koda et al (25), most malignant colorectal tumors showed intracytoplasmic staining of Cx26. In this study, however, only a subset of the adenocarcinoma cases (23.8\%) showed intracytoplasmic staining, and when compared to the level of staining for adenomas or normal epithelium, this level was significant. Therefore, loss or reduction of the intercellular expression of Cx26, and an alteration of its cellular localization as seen by intracytoplasmic staining may be associated with a role of this protein in the carcinogenic process of colorectal cancer. The low level of intracytoplasmic staining and the lack of correlation between intracytoplasmic staining and any of the clinicopathologic parameters may be explained by the subjectivity 
of the interpretation of the staining results and the limited number of samples (especially high-stage samples) that could introduce bias.

The level of tumor invasion (pT) and the loss of Cx26 expression showed a significant correlation, suggesting that Cx26 may play a role in tumor progression, particularly in tumor invasion. In contrast, expression of Cx26 and lymph node metastasis $(\mathrm{pN})$ or metastasis to other organs $(\mathrm{pM})$ were not significantly associated. Of the 130 cases, it was assumed that cases with lymph node metastasis (44 out of 130) were 41 pN1 and 3 pN2 cases. As metastasis to other organs was limited to three cases, this small sample size may have had an influence on the findings.

A postsurgical follow-up observation was performed to examine the association of the status of Cx26 expression and its localization with patient survival. No significant association was found.

Mutation of the p53 protein, a known tumor suppressor gene, is one of the most frequently observed genetic alterations in human tumors. The product of this gene is a nuclear protein that is involved in the cell cycle, apoptosis, and chromosomal stability regulation (33). Results of this study revealed that expression of p53 protein was significantly correlated to the expression of $\mathrm{Cx} 26$ on the cell membrane.

In summary, for colorectal adenocarcinomas, reduction in the intercellular Cx26 staining was associated with the depth of tumor invasion, and intracytoplasmic staining of Cx26 was observed only in adenocarcinomas. This finding suggests that the alteration of the expression of Cx26 plays a role in the formation of colorectal adenocarcinomas and their progression. Furthermore, for colorectal adenocarcinomas the loss of intercellular Cx26 staining and the expression of $\mathrm{p} 53$ protein showed a significant correlation. Therefore, intracytoplasmic staining and loss of intercellular staining of Cx26 and strong p53 staining of a colorectal adenocarcinoma suggest the presence of a high stage lesion. Additional studies will be required to examine further the role of $\mathrm{Cx} 26$ in the carcinogenic process which may be useful for patient prognosis as determined by immunohistochemical staining. Additional studies examining an association between expression of p53 and loss or reduction of Cx26 expression are also suggested.

\section{Acknowledgements}

This work was supported by the Korean Science and Engineering Foundation (KOSEF) grant funded by the Korean government (MOST) (R13-2003-009). We are grateful to Dr So-Yeon Ryu and the Medical Research Institute, Chosun University, for statistical analysis.

\section{References}

1. Charles AC, Naus CC, Zhu D, Kidder GM, et al: Intercellular calcium signaling via gap junction in glioma cells. J Biol Chem 118: 195-201, 1992.

2. Ruch RJ: The role of gap junctional intercellular communication in neoplasia. Ann Clin Lab Sci 24: 216-231, 1994.

3. Mehta PP, Lokeshwar BL, Schiller PC, Bendix MV, et al: Gapjunctional communication in normal and neoplastic prostate epithelial cells and its regulation by cAMP. Mol Carcinog 15: 18-32, 1996.
4. Ruch RJ, Guan X and Sigler K: Inhibition of gap junctional intercellular communication and enhancement of growth in BALB/c3T3 cells treated with connexin43 antisense oligonucleotides. Mol Carcinog 14: 269-274, 1995.

5. Weinstein RS, Merk FB and Alroy J: The structure and function of intercellular junctions in cancer. Adv Cancer Res 23: 23-89, 1976.

6. Yamasaki H: Gap junctional intercellular communication and carcinogenesis. Carcinogenesis 11: 1051-1058, 1990.

7. Klaunig JE, Ruch RJ, Weghorst CM and Hampton JA: Role of inhibition of intercellular communication in hepatic tumor promotion. In Vitro Toxicol 3: 91-107, 1990.

8. Ruch RJ and Klaunig JE: The effects of tumor promoters, genotoxic carcinogens, and hepatocytotoxins on mouse hepatocyte intercellular communication. Cell Biol Toxicol 2: 469-483, 1986.

9. Kamibayashi Y, Oyamada Y, Mori M and Oyamada M: Aberrant expression of gap junction proteins (connexins) is associated with tumor progression during multistage mouse skin carcinogenesis in vivo. Carcinogenesis 16: 1287-1297, 1995.

10. Jinn Y, Ichioka M and Marumo F: Expression of connexin32 and connexin43 gap junction proteins and E-cadherin in human lung cancer. Cancer Lett 127: 161-169, 1998.

11. Mourelle M, Casellas F, Guarner F, Salas A, et al: Induction of nitric oxide synthase in colonic smooth muscle from patients with toxic megacolon. Gastroenterology 109: 1492-1496, 1995.

12. Krutovskikh V, Mazzoleni G, Mironov N, Omori Y, et al: Altered homologous and heterologous gap-junctional intercellular communication in primary human liver tumors associated with aberrant protein localization but not gene mutation of connexin 32. Int J Cancer 56: 87-94, 1995.

13. Huang RP, Hossain MZ, Sehgal A and Boynton AL: Reduced connexin43 expression in high-grade human brain glioma cells. J Surg Oncol 70: 21-24, 1999.

14. Tsai H, Werber J, Davia MO, Edelman M, et al: Reduced connexin 43 expression in high grade, human prostatic adenocarcinoma cells. Biochem Biophys Res Commun 227: 64-69, 1996.

15. Oyamada Y, Oyamada M, Fusco A and Yamasaki H: Aberrant expression, function, and localization of connexins in human esophageal carcinoma cell lines with different degrees of tumorigenicity. J Cancer Res Clin Oncol 120: 445-453, 1994.

16. Defamie N, Mograbi B, Roger C, Cronier L, et al: Disruption of gap junctional intercellular communication by lindane is associated with aberrant localization of connexin 43 and zonula occludens-1 in 42GPA9 Sertoli cells. Carcinogenesis 22: 1537-1542, 2001.

17. Eghbali B, Kessler JA, Reid LM, Roy C, et al: Involvement of gap junctions in tumorigenesis: transfection of tumor cells with connexin 32 cDNA retards growth in vivo. Proc Natl Acad Sci USA 88: 10701-10705, 1991 .

18. Huang RP, Fan Y, Hossain MZ, Peng A, et al: Reversion of the neoplastic phenotype of human glioblastoma cells by connexin 43 (cx43). Cancer Res 58: 5089-5096, 1998.

19. Yamasaki H and Naus CC: Role of connexin genes in growth control. Carcinogenesis 17: 1199-1213, 1996.

20. Omori Y, Zaidan Dagli ML, Yamakage K and Yamasaki H: Involvement of gap junctions in tumor suppression: analysis of genetically-manipulated mice. Mutat Res 477: 191-196, 2001.

21. Jamieson S, Going JJ, D'Arcy R and George WD: Expression of gap junction proteins connexin 26 and connexin 43 in normal human breast and in breast tumors. J Pathol 184: 37-43, 1998.

22. Ito A, Katoh F, Kataoka TR, Okada M, et al: A role for heterologous gap junction between melanoma cells and endothelial cells in metastasis. J Clin Invest 105: 1189-1197, 2000.

23. Kinzler KW and Vogelstein B: Colorectal tumors. In: The Genetic Basis of Human Cancer. Vogelstein B and Kinzler KW (eds). McGraw-Hill, New York, pp583-612, 2002.

24. Greene FL, Page DL and Fleming ID (eds): AJCC Cancer Staging Manual. Springer-Verlag, New York, 2002.

25. Kanczuga-Koda L, Sulkowski S, Koda M and Sulkowska M: Alteration in Connexin26 expression during colorectal carcinogenesis. Oncology 68: 217-222, 2005.

26. Willecke K, Eiberger J, Degen J, Eckardt D, et al: Structural and functional diversity of connexin genes in the mouse and human genome. Biol Chem 383: 725-737, 2002.

27. Krutovskikh VA, Piccoli $\mathrm{C}$ and Yamasaki H: Gap junction intercellular communication propagates cell death in cancerous cells. Oncogene 21: 1989-1999, 2002. 
28. Trosko JE and Chang CC: Mechanism of up-regulated gap junctional intercellular communication during chemoprevention and chemotherapy of cancer. Mutat Res 219: 480-481, 2001.

29. Evans WH and Martin PE: Gap junction structure and function. Mol Membr Biol 19: 121-136, 2002.

30. Akihiko I, Yu-ichihiro K, Kazuya U, Tomoyo O, et al: Increased expression of connexin26 in the invasive component of lung squamous cell carcinoma significant correlation with poor prognosis. Cancer Lett 28: 239-248, 2006.
31. Krutovskikh VA, Troyanovsky SM, Piccoli C, Tsuda H, et al Differential effect of subcellular localization of communication impairing gap junction protein connexin43 on tumor cell growth in vivo. Oncogene 19: 505-513, 2000.

32. Olbina G and Eckhart W: Mutations in the second extracellular region of connexin 43 prevent localization to the plasma membrane, but do not affect its ability to suppress cell growth. Mol Cancer Res 1: 690-700, 2003.

33. Bruner JM, Connelly JH and Saya H: p53 protein immunostaining in routinely processed paraffin-embedded sections. Mod Pathol 6: 189-194, 1993. 